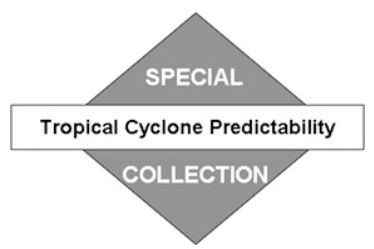

\title{
Interaction of Typhoon Shanshan (2006) with the Midlatitude Trough from both Adjoint-Derived Sensitivity Steering Vector and Potential Vorticity Perspectives
}

\author{
Chun-Chieh Wu, Shin-Gan Chen, Jan-Huey Chen, Kun-Hsuan Chou, and Po-Hsiung Lin \\ Department of Atmospheric Sciences, National Taiwan University, Taipei, Taiwan
}

(Manuscript received 6 March 2008, in final form 30 June 2008)

\begin{abstract}
Targeted observation is one of the most important research and forecasting issues for improving tropical cyclone predictability. A new parameter [i.e., the adjoint-derived sensitivity steering vector (ADSSV)] has been proposed and adopted as one of the targeted observing strategies in the Dropwindsonde Observations for Typhoon Surveillance near the Taiwan Region (DOTSTAR). The ADSSV identifies the sensitive areas at the observing time to the steering flow at the verifying time through the adjoint calculation. In this study, the ADSSV is calculated from the nonlinear forecast model of the fifth-generation Pennsylvania State University-National Center for Atmospheric Research (PSU-NCAR) Mesoscale Model (MM5) and its adjoint to interpret the dynamical processes in the interaction between Typhoon Shanshan (2006) and the midlatitude trough. The ADSSV results imply that high-sensitivity regions affecting the motion of Typhoon Shanshan are located at the edge of the subtropical high and the 500-hPa midlatitude trough over northern central China. These ADSSV signals are in very good agreement with the quantitative evaluation based on the potential vorticity (PV) diagnosis. The vertical structure of the ADSSV is also shown for more physical insights into the typhoon-trough interaction. The maximum ADSSV occurs at $800-500 \mathrm{hPa}$ to the southeast of Shanshan (associated with the subtropical high), while distinct ADSSV signals are located upstream of the storm center at about 500-300 hPa (associated with the mid- to upper-tropospheric midlatitude trough). Overall, it is demonstrated that the ADSSV features can well capture the signal of the large-scale trough feature affecting the motion of Shanshan, which can also be well validated from the PV analysis.
\end{abstract}

\section{Introduction}

Conventional observational data are inadequate in providing accurate initial and boundary conditions for numerical model simulations and forecasts of tropical cyclones (TCs), thus often leading to poor track and intensity forecasts (Wu and Kuo 1999; Wu et al. 2005). Therefore, making additional observations in the critical areas that will have the maximum influence on numerical forecasts of TCs is an important task. Operational aircraft surveillance missions have been conducted by the Tropical Prediction Center (TPC) and the Hurricane Research Division (HRD) on Atlantic hurricanes since 1997 (Aberson 2003), and also by the Dropwindsonde Observations for Typhoon Surveillance near the Taiwan Region (DOTSTAR) project (Wu et al.

Corresponding author address: Dr. Chun-Chieh Wu, Dept. of Atmospheric Sciences, National Taiwan University, No. 1, Sec. 4, Roosevelt Rd., Taipei 106, Taiwan.

E-mail: cwu@typhoon.as.ntu.edu.tw
2005) on western North Pacific typhoons since 2003. Wu et al. (2007a) have demonstrated that the dropwindsonde data of DOTSTAR have positive impacts on TC track forecasts of four models: the National Centers for Environmental Prediction (NCEP) Global Forecast System (GFS), the Navy Operational Global Atmospheric Prediction System (NOGAPS) of the Fleet Numerical Meteorology and Oceanography Center (FNMOC), the Japanese Meteorological Agency (JMA) Global Spectral Model (GSM), and the Weather Research and Forecasting (WRF) model.

Because of the limited resources from aircraft observations, the targeted observation strategy, involving the optimal design of the flight route and locations for dropwindsondes deployment, has to be further developed for typhoon surveillance missions in order to significantly improve numerical forecasts. Targeted observation has been an active research topic in numerical weather prediction with plans for field programs, tests of new observing systems, and application of new concepts in predictability and data assimilation (Langland 2005). 
In addition, targeted observation is one of the highlighted issues recommended by World Meteorological Organization's (WMO's) Sixth International Workshop on Tropical Cyclones (IWTC-VI) in 2006 (Wu 2006). The prerequisite for devising the observing strategy is to identify the sensitive areas that will have the greatest influence on improving the numerical forecast, or minimizing the forecast errors.

A new parameter, adjoint-derived sensitivity steering vector (ADSSV), has been proposed (Wu et al. 2007b) and adopted as one of the targeted observing strategies in DOTSTAR. By appropriately defining the response functions (see section 2a), the ADSSV represents the sensitivity of the mean steering flow at the verifying time to the flow field at the observing time through the adjoint calculation (Zou et al. 1997). While identifying the sensitive areas affecting the TC motion, $\mathrm{Wu}$ et al. (2007b) selected Typhoons Meari and Mindulle (2004) to show the validity of ADSSV and found that the forecasts of their tracks, in some degrees, are sensitive to the edge of the subtropical high. In addition, they also identified signals of the binary interaction between Typhoons Fengshen and Fungwong (2002) using the ADSSV method. They showed that Typhoon Fengshen is sensitive to the steering flow associated with the circulation of Fungwong, but the sensitivity of Fungwong to the steering flow of Fengshen is rather insignificant, which is consistent with the potential vorticity (PV) analysis of Yang et al. (2008), implicating the nature of the one-way interaction between the two typhoons.

In addition to the flows associated with the subtropical high and the binary interaction between two TCs that could affect the track of TCs, the steering due to the midlatitude trough may also play an important role in causing TCs to recurve poleward into the high latitude. The purpose of this study is to examine how the influence from the large-scale trough feature can be captured by the ADSSV method and to interpret the dynamics and interactions between TCs and the midlatitude trough from the signals acquired from the ADSSV. On the other hand, the PV diagnosis (Wu et al. 2003, 2004) is also employed to interpret and verify the physical meaning of the TC-trough interaction. Section 2 briefly reviews the methodology of the ADSSV and the PV inversion. The results of the typhoon-trough interaction based on the ADSSV and PV analyses are shown in section 3 , and the summary is presented in section 4 .

\section{Methodology and experimental design}

\section{a. $A D S S V$}

One of the techniques to identify the locations for adaptive observations in order to improve weather forecast of specific interests is to recognize the sensitivity of the specific model output to the initial input variables. Errico (1997) has shown that the adjoint model can be a powerful tool for many studies that require an estimate of sensitivity of model output with respect to input. In other words, by defining the response function, as a function of model output variables, and of research interests, one can use the adjoint model to effectively calculate the sensitivity of the response function to model input variables (e.g., $\partial R / \partial \mathbf{x}_{\text {in }}$; Kleist and Morgan 2005).

The response functions are defined (Wu et al. 2007b) as the deep-layer-mean wind within the verifying area that is a square of $600 \mathrm{~km} \times 600 \mathrm{~km}$ centered around the fifth-generation Pennsylvania State UniversityNational Center for Atmospheric Research (PSU-NCAR) Mesoscale Model (MM5)-simulated storm location at the verifying time. The two response functions are $R_{1}$, the deep-layer area average wind (Wu et al. 2003) of the zonal component $(u)$ between 0.875 and 0.225 sigma levels (roughly between 850 and $250 \mathrm{hPa}$ ); and $R_{2}$, the average of the meridional component $(v)$ of the wind vector:

$$
R_{1} \equiv \frac{\int_{0.875}^{0.225} \int_{A} u d x d y d \sigma}{\int_{0.875}^{0.225} \int_{A} d x d y d \sigma} \text { and } R_{2} \equiv \frac{\int_{0.875}^{0.225} \int_{A} v d x d y d \sigma}{\int_{0.875}^{0.225} \int_{A} d x d y d \sigma}
$$

In the adjoint model, the integration is completed by summing up the zonal $(u)$ and meridional $(v)$ winds on the even-interval sigma coordinate between $\sigma=0.875$ and 0.225 within the verifying area. In other words, the vector of $\left(R_{1}, R_{2}\right)$ represents the background steering flow above the storm center at the verifying time.

First, $d R_{1} / d u$ and $d R_{1} / d v$ (as well as $d R_{2} / d u$ and $d R_{2} / d v$ ) are calculated to indicate the sensitivity of the zonal (meridional) component of the steering flows at the verifying time to the flow fields at the observing time. As indicated in $\mathrm{Wu}$ et al. (2007b), instead of looking at the sensitivity (gradient) of $R$ to $u$ and $R$ to $v$ (i.e., $\partial R / \partial u$ and $\partial R / \partial v$ ), a parameter, ADSSV $(\partial R / \partial s$ and $\partial R / \partial D$, where $s$ and $D$ represent the vorticity and the divergence fields, respectively), is designed to identify the sensitive areas at the observing time to the steering flow at the verifying time. Note that since the large-scale wind field affecting the TC track is more likely to be nondivergent, it has been demonstrated in Wu et al. (2007b) that $\partial R / \partial s$ is the dominant term as compared to $\partial R / \partial D$. Therefore, we identify the $\partial R / \partial s$ as the essential metric to represent the ADSSV. In other words, the ADSSV with respect to the vorticity field can be shown as

$$
\mathrm{ADSSV} \equiv\left(\frac{\partial R_{1}}{\partial \varsigma}, \frac{\partial R_{2}}{\partial \varsigma}\right)
$$


where at a given point, the magnitude of ADSSV indicates the extent of the sensitivity. Meanwhile, the direction of the ADSSV represents the direction toward which the steering flow would increase at the verifying time with respect to a vorticity perturbation placed at that point at the observing time.

\section{b. PV diagnosis}

The PV invertibility principle states that given a distribution of PV, a prescribed balanced condition, and boundary conditions, the balanced mass and wind fields can be recovered. Another robust strength associated with inversion of PV is the so-called piecewise PV inversion; that is, when the flow field is appropriately divided into the mean and perturbation components, the balanced fields associated with each individual PV perturbation could be obtained from the perturbation PV equations (Davis 1992). Wu and Emanuel (1995a,b) applied such a method to evaluate the influence of the large-scale environment and the different PV features on the storm's track.

To understand how the environmental flows affected the typhoon, the axisymmetric average relative to the center of the typhoon is taken as the mean part and the rest as the perturbation field, and then the approach by Shapiro (1996) and Wu et al. (2003) is followed to perform the piecewise PV inversion. In other words, the azimuthal average of the wind field is first constructed to obtain the average streamfunction $(\bar{\Psi})$, so that the associated average geopotential height $(\hat{\Phi})$ and average PV field $(\hat{q})$ can be derived from the nonlinear balance equation (Charney 1955) and the Ertel PV equation on $\pi\left[\pi=C_{p}\left(p / p_{0}\right)^{\kappa}\right]$ and spherical coordinates, respectively. By taking the total perturbation field as $\Psi^{\prime}=\Psi-\bar{\Psi}, \Phi^{\prime}=\Phi-\hat{\Phi}$, and $q^{\prime}=q-\hat{q}$, the piecewise PV inversion is performed to calculate the balanced flow and mass fields associated with each PV perturbation. More detailed methodology of PV inversion can be found in Wu et al. $(2003,2004)$ and Yang et al. (2008). To investigate how the flow field associated with the trough and the subtropical high affected the motion of Typhoon Shanshan, the PV perturbations associated with the trough $\left(q_{\mathrm{TR}}^{\prime}\right)$ and the subtropical high $\left(q_{\mathrm{SH}}^{\prime}\right)$ are demonstrated in this study.

\section{c. Experimental design}

This study utilizes the MM5 adjoint modeling system (Zou et al. 1997; Kim and Jung 2006; Wu et al. 2007b), which includes the nonlinear MM5, its tangent linear model (TLM), and a corresponding dry-physics adjoint model. The domain for the nonlinear and adjoint models used is a $60-\mathrm{km}$ resolution, $85 \times 115$ (latitude by longitude) horizontal grid points, with 20 even-interval sigma levels in the vertical. The physical parameterizations of the nonlinear integrations include the Grell cumulus parameterization, the bulk PBL scheme, the simple cooling radiation scheme, and the stable precipitation in which the large-scale saturation is removed and rained out immediately but without rain evaporation or explicit cloud prediction. The same parameterizations (or their adjoints) are applied in the TLM and adjoint integrations, but the effect of moisture is neglected. The forward and backward integrations were executed by the MM5 forecast model and the adjoint model, respectively, to calculate the sensitivity of the response function to model input variables. The initial and boundary conditions of the MM5 model and the data for PV diagnosis are acquired from the NCEP GFS global analysis $\left(1^{\circ} \times 1^{\circ}\right)$.

For Typhoon Shanshan, 0000 UTC on three successive days from 14 to 16 September 2006 are set as the observing times (denoted as EXP1, EXP2, and EXP3, respectively) and $48 \mathrm{~h}$ after each observing time as the verifying times (Table 1 ). Note that unlike the real-time operation of targeted observations (such as in DOTSTAR) where typically 48 -h forecast lead time would be needed, for simplicity and as a first-order approximation, no forecast lead time is applied here (it can be shown that the sensitivity patterns remain consistent when experiments with 48-h lead time are performed; figures not shown). These experiments are conducted to investigate whether the ADSSV features would pick up some early signals of the typhoon-trough interaction. Because the midlatitude troughs mostly occur near 500 $\mathrm{hPa}$, the ADSSV with respect to the 500-hPa vorticity field is demonstrated in this study. In addition, the PV analysis of the 6-hourly NCEP GFS global analysis data from 0000 UTC 14 September to 0000 UTC 18 September is conducted based on the PV diagnosis method (Wu et al. 2003, 2004), within the selected domain of $7^{\circ}-52^{\circ} \mathrm{N}, 95^{\circ}-155^{\circ} \mathrm{E}$.

\section{Results}

\section{a. Synopsis}

The best track of Typhoon Shanshan from the Central Weather Bureau (CWB) in Taiwan and the modelsimulated tracks of three experiments (i.e., EXP1, EXP2, and EXP3) are shown in Fig. 1. Typhoon Shanshan formed to the west of Guam at 1200 UTC 10 September 2006, then moved northwestward before intensifying and reaching the scale of a moderate typhoon at 1800 UTC 11 September. Over the next two days, the moving direction of Shanshan gradually turned to the west. Shanshan was located between the midlatitude trough over northern-central China and the 
TABLE 1. Observing and verifying times for three experiments.

\begin{tabular}{ccc}
\hline \hline Expt name & Observing time & Verifying time \\
\hline EXP1 & 0000 UTC 14 Sep 2006 & 0000 UTC 16 Sep 2006 \\
EXP2 & 0000 UTC 15 Sep 2006 & 0000 UTC 17 Sep 2006 \\
EXP3 & 0000 UTC 16 Sep 2006 & 0000 UTC 18 Sep 2006 \\
\hline
\end{tabular}

subtropical high, which is divided into two areas by the midtropospheric nearly cutoff low near $25^{\circ} \mathrm{N}, 145^{\circ} \mathrm{E}$ (Fig. 2a). On around 15 September, the translation speed of Shanshan became slower and it changed its direction to the north, passing through the ocean to the east of Taiwan. The subtropical high remained to the east of Shanshan while the trough slightly deepened and moved southeastward, located upstream of Shanshan (Fig. 2b). Then, Shanshan started to accelerate northeastward on 16 September as it began to interact with a midlatitude trough over eastern China (Fig. 2c). The system began to weaken and experienced the extratropical transition on 17 September when it went northnortheastward toward Japan, as indicated in Figs. 2d,e.

\section{b. ADSSV for the typhoon-trough interaction}

The ADSSV with respect to vorticity, as indicated in Eq. (2), is used to represent the sensitivity of the typhoon steering flow at the verifying time to the vorticity field at the observing time. The ADSSV results with respect to the $500-\mathrm{hPa}$ vorticity field at the observing time for three experiments are shown in Fig. 3, superposed with the $500-\mathrm{hPa}$ geopotential height. The negative sign before $48 \mathrm{~h}$ indicates the backward integration (using the negative time step) associated with the adjoint model. The magnitude of ADSSV has been normalized by the maximum value in the domain to clearly identify the relative sensitivity. In EXP1, the ADSSV at the observing time (0000 UTC 14 September; Fig. 3a) shows that the major sensitive regions are located around Typhoon Shanshan at distances ranging from 400 to $1000 \mathrm{~km}$, with the maximum sensitivity to the southeast of the center and the local maxima to the northwest. Note that most ADSSVs are distributed in the subtropical high region (as indicated by the 500 -hPa geopotential height in Fig. 3a), implying that the information of the surrounding subtropical high is important to the track of Shanshan at the verifying time. However, there is no sign of ADSSV features associated with the midlatitude trough around this time.

Figure $3 \mathrm{~b}$ shows the ADSSV at the observing time (0000 UTC 15 September) for EXP2. Obviously, two major ADSSV features can be identified. One is located at about $800 \mathrm{~km}$ to the southeast of Shanshan, and the other occurs in the midlatitude trough region at $500 \mathrm{hPa}$ near northern-central China. It is indicated that in ad-

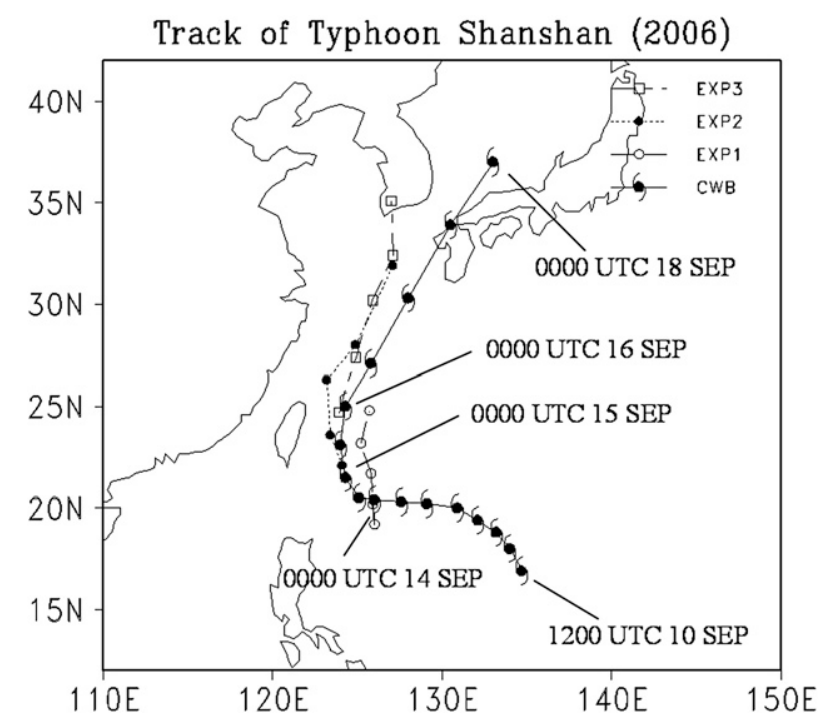

FIG. 1. The CWB best track (typhoon symbols) and the MM5 48-h forecast tracks of Typhoon Shanshan in the three experiments: EXP1, EXP2, and EXP3. Each symbol is plotted at 12-h intervals.

dition to the high sensitivity associated with the edge of the subtropical high, the motion of Typhoon Shanshan is also sensitive to the flow associated with the trough at far northwest of the storm. In other words, the variation of the vorticity field associated with the trough at the observing time would likely lead to the track variation of Shanshan at the verifying time. Note that the vectors in the trough region mainly point northward, implicating that the northward component of steering flow at the verifying time would strengthen (weaken) if the vorticity in the trough area increases (decreases) at the observing time. In addition, some sensitivity occurs near the center of Shanshan, indicating that the vorticity variation associated with TC itself would likely affect the TC track. Nevertheless, this appears as a minor sensitivity as compared to other regions with higher values (e.g., near the edge of the subtropical high and the midlatitude trough).

The evolution of ADSSV integrated backward to different forecast times by the adjoint model for EXP2 is shown in Fig. $4(-12,-24$, and -36 h) and Fig. $3 b$ $(-48 \mathrm{~h})$, in which the corresponding geopotential height at $500 \mathrm{hPa}$ is also superposed. These ADSSV signals represent the sensitive areas at the different forecast times, which will affect the steering flow of Shanshan at the verifying time. Following the integration, it is found that a portion of distinct ADSSV features propagates along with the axis of the midlatitude trough and finally moves into the verifying area, indicating that the steering flow of Shanshan at the verifying time is likely to be affected by the flow associated with the trough. 
(a) 0000 UTC 14 SEP 2006

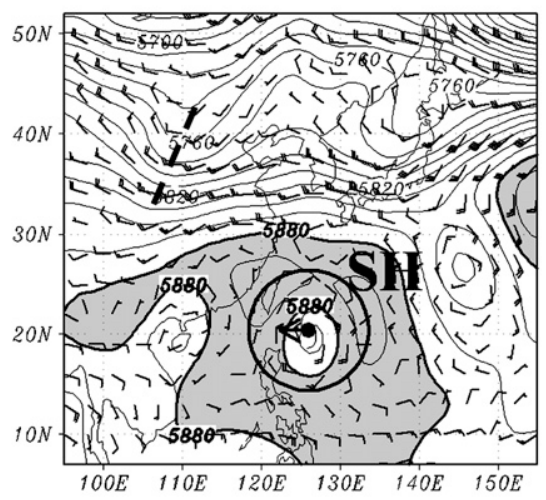

(d) 0000 UTC 17 SEP 2006

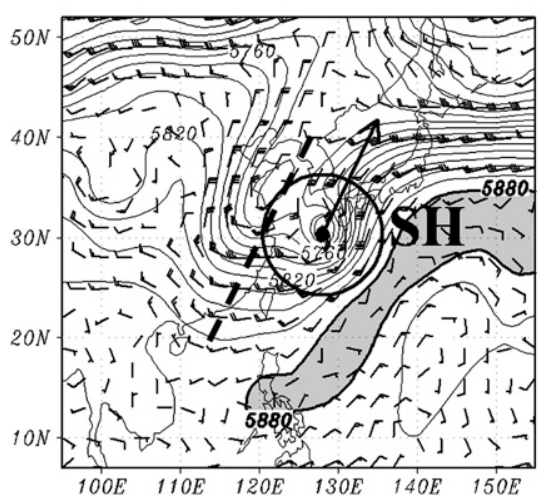

(b) 0000 UTC 15 SEP 2006

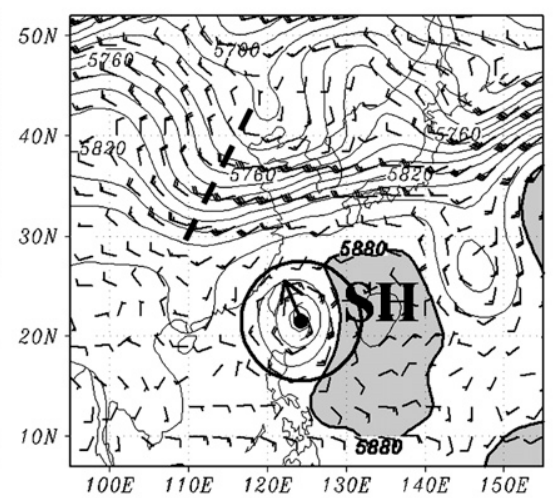

(e) 0000 UTC 18 SEP 2006 (c) 0000 UTC 16 SEP 2006

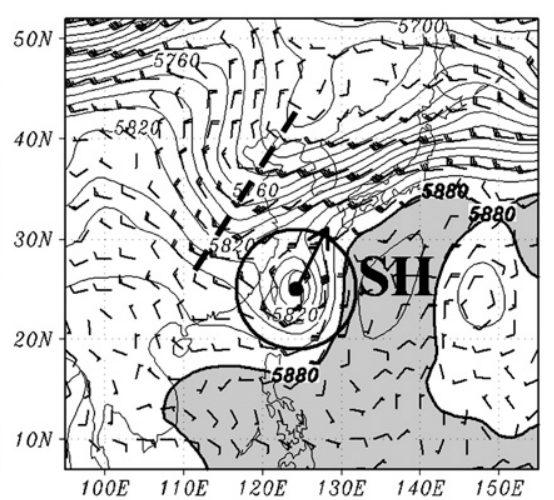

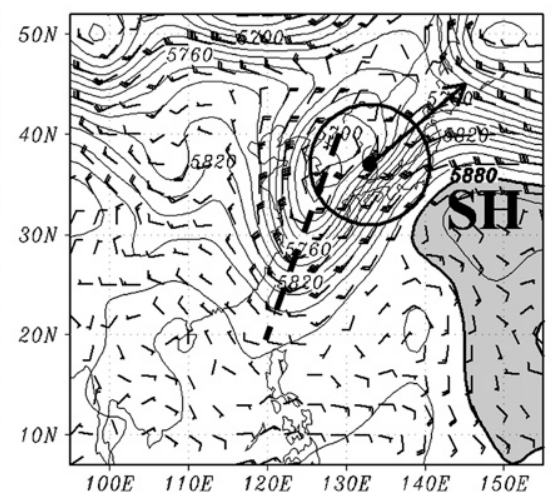

FIG. 2. Geopotential height (contour interval of $15 \mathrm{gpm}$; the area with values larger than $5880 \mathrm{gpm}$ is shaded) and wind at $500 \mathrm{hPa}$ (one full wind barb $=5 \mathrm{~m} \mathrm{~s}^{-1}$ ) starting from (a) 0000 UTC 14 Sep to (e) 0000 UTC 18 Sep 2006 at 24-h intervals. The instantaneous movement of Shanshan is indicated by the thick black arrow, the length of which represents the actual translation velocity, and the circle shows the scale of $5 \mathrm{~m} \mathrm{~s}^{-1}$. The midlatitude trough and the subtropical high are roughly indicated by the dashed line and the symbol "SH," respectively.

To gain more physical insights, the vertical structure of the ADSSV at the observing time for EXP2 is examined (Fig. 5). The two circles of radii measuring 800 and $2100 \mathrm{~km}$ each centered at the storm position at the observing time (Fig. 3b) are unfolded along the clockwise (i.e., south-west-north-east) direction to investigate the vertical structure of ADSSV. We choose the 2100-km radius for the purpose of including the area of the trough near northern-central China. The magnitude of ADSSV is normalized by the maximum value at the level of $500 \mathrm{hPa}$. At $800 \mathrm{~km}$ away from the storm center (Fig. 5a), the maximum ADSSV occurs at 700-500 $\mathrm{hPa}$ in the middle and lower troposphere to the southeast of the storm center. This sensitive area is mainly related to the edge of the subtropical high indicated by the geopotential height in Fig. 3b. The vertical cross section of the vorticity field at $2100 \mathrm{~km}$ far distant from Shanshan (Fig. 5b) shows that there is positive vorticity accompanied with the trough in the middle and upper troposphere to the northwest of the storm center. Also, some
ADSSV signals exist close to this positive vorticity above the level of $500 \mathrm{hPa}$. As expected, it is indicated that the change of Shanshan's steering flow at the verifying time would be induced partially from the variation in vorticity associated with the midlatitude trough.

At 0000 UTC 16 September 2006, the midlatitude trough is located upstream of Typhoon Shanshan and the storm gradually heads north-northeast to Japan (Figs. 1 and 2c). In EXP3, the result (Fig. 3c) shows that most ADSSVs are distributed in a belt region around the southern side of the storm, and clear sensitivity signals are again present in the midlatitude trough area near the central part of eastern China. It is worth noting that the relative magnitude of ADSSV sensitivity associated with the trough increases as much as that associated with the edge of the subtropical high. Also, the evolution of ADSSV for EXP3 (figure not shown) reveals that some ADSSV signals still propagate along with the trough, similar to the features in Fig. 4. This indicates that the flow of the trough may still play an 


\section{ADSSV(VOR), Geop.Height(shaded) at 500hPa}
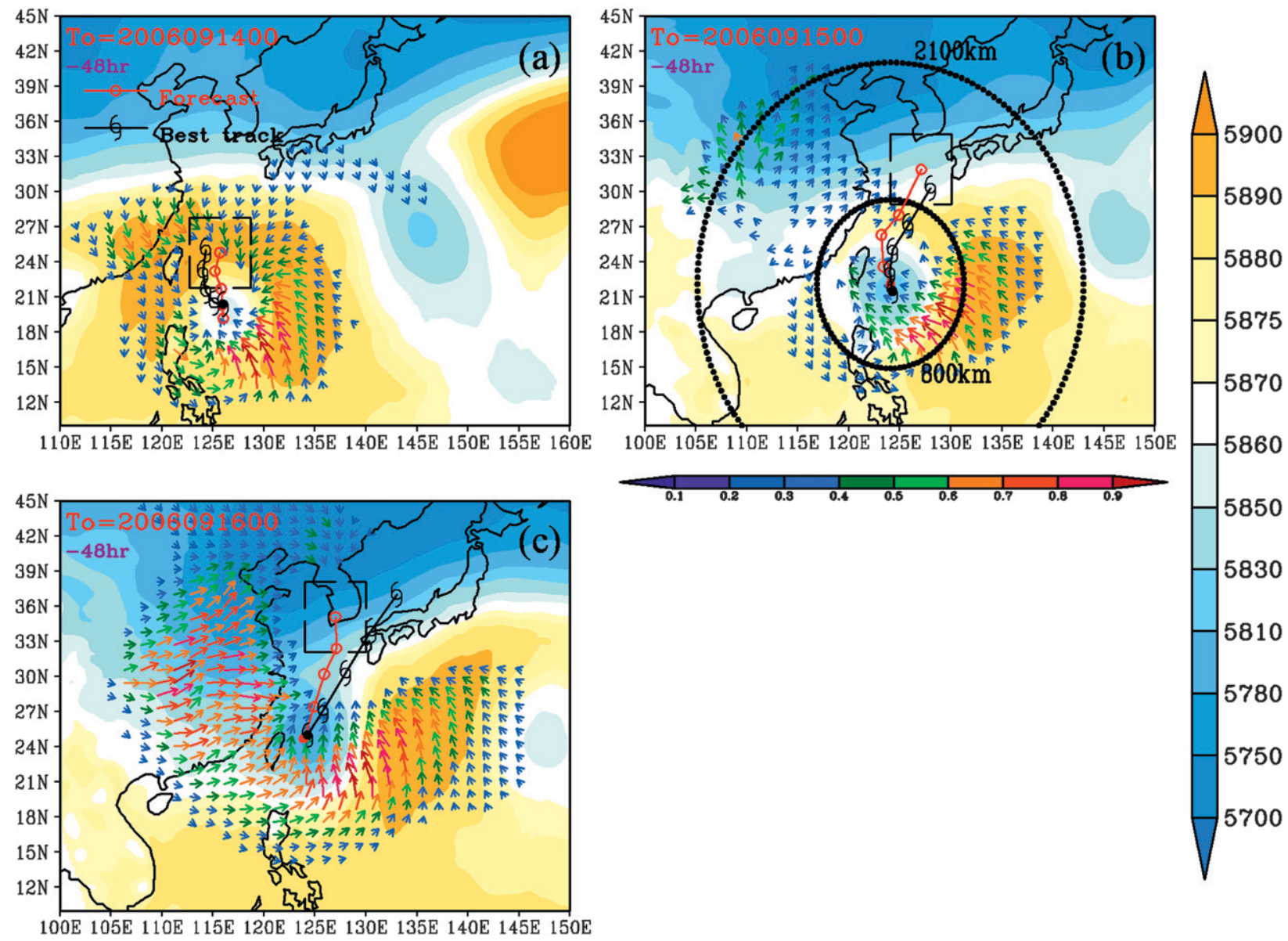

FIG. 3. ADSSV with respect to the vorticity field at $500 \mathrm{hPa}$ at the observing time, superposed with the geopotential height (magnitude scaled by the color bar to the right; $\mathrm{m}$ ) at $500 \mathrm{hPa}$ for (a) EXP1, (b) EXP2, and (c) EXP3. The magnitude of the ADSSV is normalized by the maximum value in the domain (the value smaller than 0.25 is omitted). The best track from CWB analysis and 48-h model-predicted track are indicated with the black typhoon symbols and the red circles for every $12 \mathrm{~h}$, respectively. The dashed square box represents the verifying area at the verifying time.

important role in affecting the steering flow of Shanshan at the verifying time. Meanwhile, the 500-hPa geopotential height field superposed on Fig. 3c shows that Typhoon Shanshan tends to merge with the midlatitude trough and undergoes the process of extratropical transition.

Two main concerns related to the adjoint sensitivity (i.e., the dry physical process and the linear assumption employed in the TLM) are noted here. As addressed in Wu et al. (2007b), the linear assumption in the TLM has been demonstrated to be valid up to $48 \mathrm{~h}$ in the dryphysics model by comparing initial perturbations that evolve linearly via the TLM based on the dry basic state with the difference obtained from two nonlinear model runs under the dry physics. Similar results are also obtained in this case, showing that the time evolution of wind perturbation in the TLM is almost identical to the difference from two nonlinear model runs (figures not shown). On the other hand, to evaluate the impact of the moist physics, another experiment is conducted based on the moist basic state in the TLM and the nonlinear model with moist physics. However, after the 24-h integration, the difference between two nonlinear model simulations shows many noises occurring on several spots of the model domain, which is very different from the wind perturbation in the TLM (figures not shown). This pattern is similar to that noted in $\mathrm{Wu}$ et al. (2007b). But the above comparison based on the moist basic state is actually not fair because the TLM used contains no moist physics whereas the nonlinear model has moist physics. As noted in Peng and Reynolds (2006), Wu et al. (2007b), and Kim and Jung (2009), TC movement is mainly controlled by the large-scale flow 


\section{Evolution of ADSSV(VOR) and Geop.Height(shaded)}
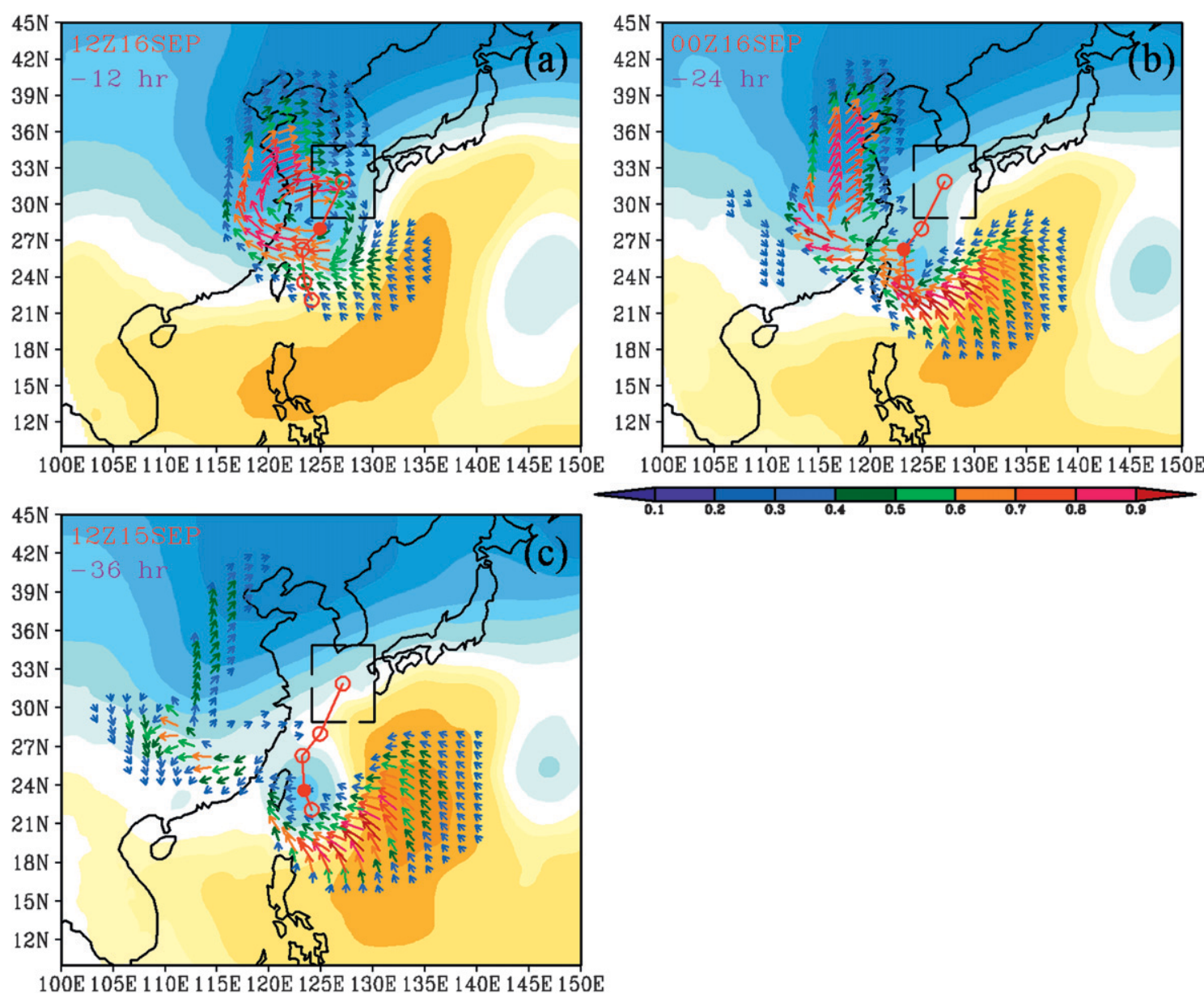

5900

5890

5880

5875

5870

5860

5850

5830

5810

5780

5750

5700

FIG. 4. The evolution of the ADSSV (magnitude of the vector is normalized by the maximum in the domain and the value smaller than 0.25 is omitted) with respect to the vorticity field at $500 \mathrm{hPa}$ for EXP2 at (a) -12, (b) - 24, and (c) $-36 \mathrm{~h}$ in the adjoint model, superposed with the 500-hPa geopotential height. The model-predicted track is indicated with the circles in red for every $12 \mathrm{~h}$, and the solid circles represent the typhoon center at the corresponding forecast times.

field that is less directly affected or dominated by the moist physics. Therefore, we believe that the use of the dry-adjoint model in this work should provide adequate sensitivity information. Nevertheless, the detailed impact of the moist-physics adjoint model remains to be further explored in the future.

\section{c. PV diagnosis}

Judging from the ADSSV signals as shown in the previous section, the track variation of Typhoon Shanshan is mainly sensitive to the flows associated with the subtropical high and the midlatitude trough. In this section, the PV diagnosis, as described in section $2 \mathrm{~b}$, is employed to assess whether the sensitive areas picked up by the ADSSV would actually affect the TC motion at the verifying time. In other words, the PV diagnosis is applied to examine the steering effect from the subtropical high and the trough, as well as the validity of those signals from the ADSSV analysis.

The total PV perturbation $\left(q^{\prime}\right)$ and the steering flow $\left(\mathbf{V}_{\text {SDLM }}\right)$, defined as the deep-layer-mean $(925-300 \mathrm{hPa})$ wind vector averaged over the inner $3^{\circ}$ latitude around the storm center (see Wu et al. 2003), are shown in Fig. 6. In Fig. 6, the mean PV associated with Shanshan has been removed (as defined in section $2 b$ ). Therefore, there is no PV signal from Shanshan in the PV perturbation field. On the other hand, Fig. 6 clearly shows that the PV perturbation associated with the trough strengthens with time, with its maximum increasing from about 0.4 PVU (Fig. 6a; $1 \mathrm{PVU} \equiv 10^{-6} \mathrm{~m}^{2} \mathrm{~s}^{-1} \mathrm{~K}$ $\mathrm{kg}^{-1}$ ) to more than 1 PVU (Fig. 6e). Based on the PV 
Vertical profile of ADSSV at $r=800 \mathrm{~km}$ and vorticity

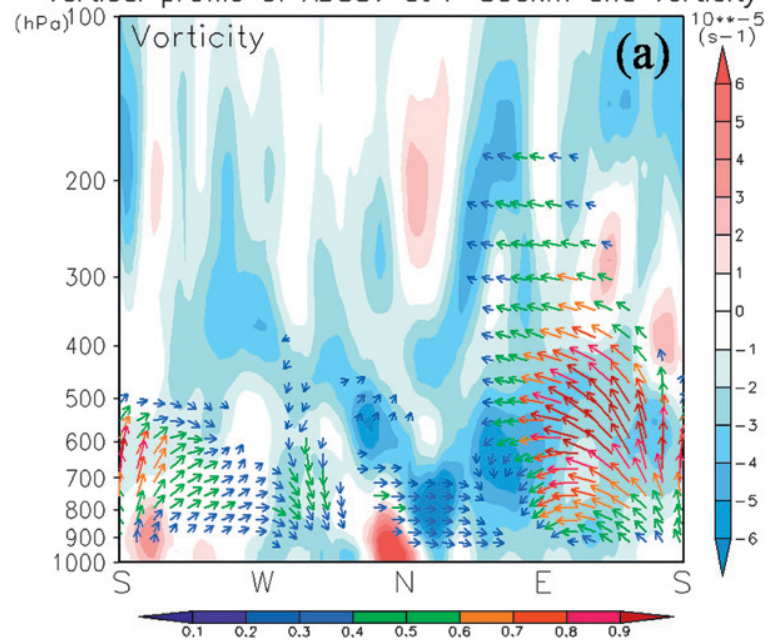

Vertical profile of ADSSV at $r=2100 \mathrm{~km}$ and vorticity

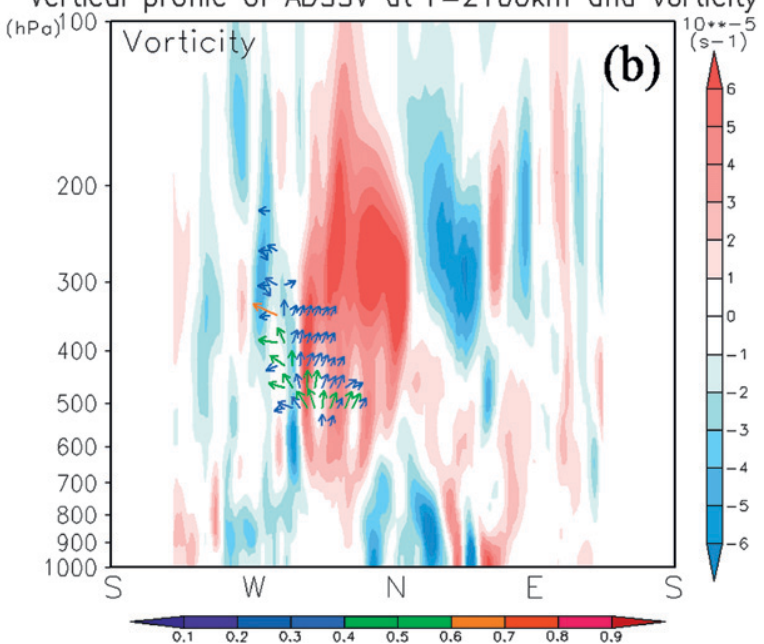

FIG. 5. For EXP2, the vertical cross section of the ADSSV on the unfolded circles indicated in Fig. 3b with the north (N) side in the center of each panel at (a) 800 and (b) $2100 \mathrm{~km}$ from the storm center. The magnitude of the ADSSV is normalized by the maximum value at $500 \mathrm{hPa}$ (the value smaller than 0.25 is omitted) and the shading represents the vorticity field (scaled by the color bar to the right: $\left.1 \times 10^{-5} \mathrm{~s}^{-1}\right)$. The vertical pressure coordinate is in logarithmic scale.

inversion indicated in section $2 \mathrm{~b}$, an example time $(0000$ UTC 17 September) is chosen to demonstrate the PV perturbation and the steering flow associated with the trough $\left(q_{\mathrm{TR}}^{\prime}\right.$, Fig. $\left.7 \mathrm{a}\right)$ and the subtropical high $\left(q_{\mathrm{SH}}^{\prime}\right.$, Fig. $7 b)$. The balanced flow fields in Fig. 7 indicate that the motion of Shanshan was almost parallel to the steering wind associated with both the trough and the subtropical high at this time.

In Fig. 8, the time series of the deep-layer-mean steering flow associated with PV perturbations of the trough and the subtropical high are compared with the actual movement of Shanshan [estimated from the 12-h best-track positions (X), i.e., $\mathbf{V}_{\mathrm{BT}}=\left(\mathbf{X}_{t+6 \mathrm{~h}}-\mathbf{X}_{t-6 \mathrm{~h}}\right) /$ 12h]. The comparison of the wind barbs in the bottom two rows in Fig. 8 shows that the balanced flow associated with the total PV perturbation $\left[\mathbf{V}_{\text {SDLM }}\left(q^{\prime}\right)\right]$ is in general agreement with the motion of the best track $\left(\mathbf{V}_{\mathrm{BT}}\right)$ except for some slight differences before 1200 UTC 15 September, indicating that the motion of Shanshan was well represented by the deep-layer-mean steering wind field in the GFS analysis based on the PV diagnosis. It is found in Fig. 8 that the steering flow associated with the PV perturbation of the trough $\left[\mathbf{V}_{\text {SDLM }}\left(q_{\mathrm{TR}}^{\prime}\right)\right]$ is slower than $5 \mathrm{~m} \mathrm{~s}^{-1}$ and the wind direction is nearly perpendicular to the flow associated with the total PV perturbation $\left[\mathbf{V}_{\text {SDLM }}\left(q^{\prime}\right)\right]$ before 0000 UTC 16 September, indicating that the trough has limited influence on the motion of Shanshan before this time. Nevertheless, a comparison between the steering flow associated with $q_{\text {TR }}^{\prime}$ and $q^{\prime}$ indicates that the steering effect of the trough is getting significant after around 1200 UTC 16 September. On the contrary, the steering flow associated with the PV perturbation of the subtropical high $\left[\mathbf{V}_{\mathrm{SDLM}}\left(q_{\mathrm{SH}}^{\prime}\right)\right]$ remains important to advect Shanshan at more than $4 \mathrm{~m} \mathrm{~s}^{-1}$. It is interesting to note that the direction of the steering flow associated with both $q_{\mathrm{TR}}^{\prime}$ and $q_{\mathrm{SH}}^{\prime}\left[\mathbf{V}_{\mathrm{SDLM}}\left(q_{\mathrm{TR}+\mathrm{SH}}^{\prime}\right)\right]$ is fairly close to the actual motion of Shanshan after 0000 UTC 15 September, indicating their dominant role in controlling the movement of Shanshan.

Following Wu et al. (2003), the value of "AT" has been calculated to quantitatively measure the influence of the steering flow associated with each PV perturbation in the direction parallel to the flow associated with the total PV perturbation. Namely, AT for the PV perturbation associated with the trough is defined as

$$
\operatorname{AT}\left(q_{\mathrm{TR}}^{\prime}\right)=\frac{\mathbf{V}_{\mathrm{SDLM}}\left(q_{\mathrm{TR}}^{\prime}\right) \cdot \mathbf{V}_{\mathrm{SDLM}}\left(q^{\prime}\right)}{\left|\mathbf{V}_{\mathrm{SDLM}}\left(q^{\prime}\right)\right|^{2}}
$$

A value of 1 means that the along-track component of the steering flow induced by the PV perturbation is identical to the flow associated with the total PV perturbation. The time evolution of AT for $q_{\mathrm{TR}}^{\prime}$ and $q_{\mathrm{SH}}^{\prime}$ is shown in Fig. 9. It is clearly found that $\operatorname{AT}\left(q_{\mathrm{SH}}^{\prime}\right)$ is always above 0.5 , whereas $\operatorname{AT}\left(q_{\mathrm{TR}}^{\prime}\right)$ ranges from 0 to -0.2 before 1200 UTC 15 September and increases to higher than 0.5 after 0600 UTC 16 September.

Note that the ADSSV at the observing time (0000 UTC 15 September) for EXP2 (Fig. 3b) also shows two major signals associated with the midlatitude trough and the subtropical high. In other words, the variation in 
(a) 0000 UTC 14 SEP 2006

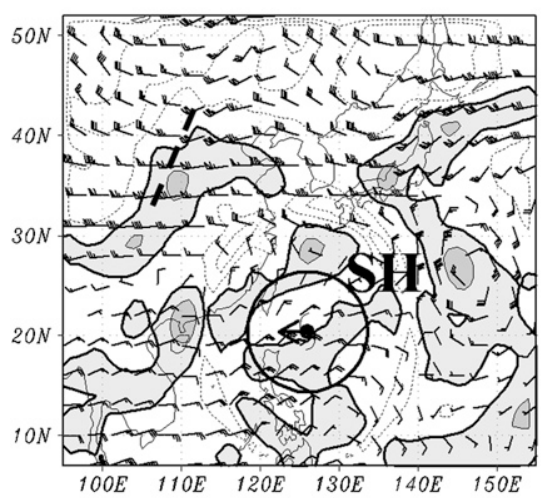

(d) 0000 UTC 17 SEP 2006 (b) 0000 UTC 15 SEP 2006

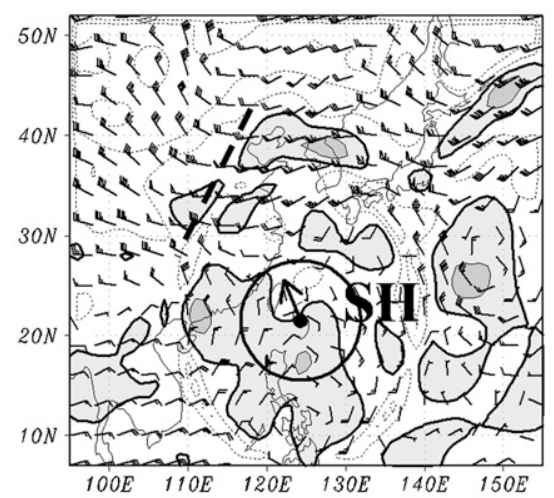

(e) 0000 UTC 18 SEP 2006 (c) 0000 UTC 16 SEP 2006

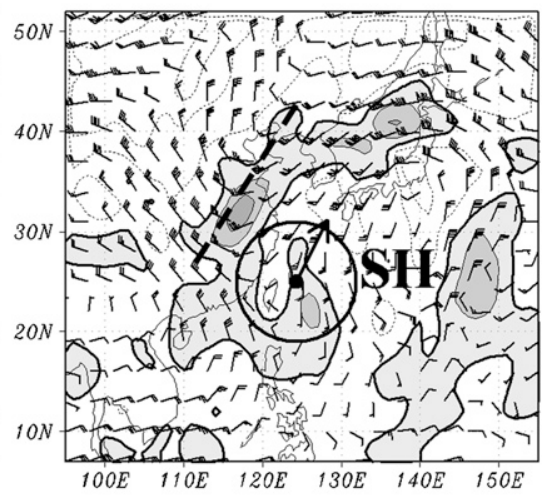

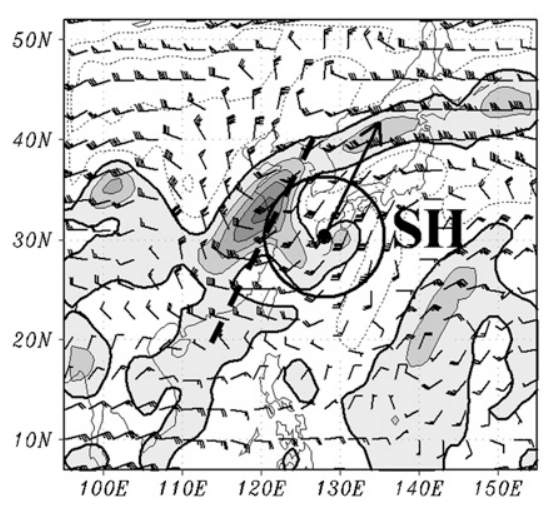

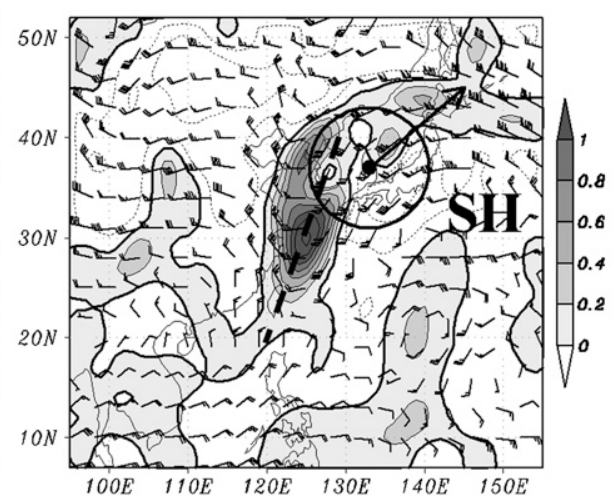

FIG. 6. Potential vorticity perturbation ( $q^{\prime}$; scaled in PVU; the positive PV perturbation is shaded) at 500 hPa, and the 925-300-hPa deep-layer-mean steering wind (one full wind barb $=5 \mathrm{~m} \mathrm{~s}^{-1}$ ) starting from (a) 0000 UTC 14 Sep to (e) 0000 UTC 18 Sep 2006 at $24-\mathrm{h}$ intervals. The definitions of the arrow and circle as well as the locations of the dashed line and symbol "SH" are as in Fig. 2.

vorticity associated with the trough and/or the subtropical high would lead to the change in the steering flow of Shanshan at the verifying time (0000 UTC 17 September). The PV diagnosis (Figs. 8 and 9) indicates that the trough and the subtropical high equally contribute to the steering flow of Shanshan at 0000 UTC 17 September, in accord with the ADSSV signals in Fig. $3 b$. In this case, the PV diagnosis confirms the steering (a) 0000 UTC 17 SEP 2006 (TR)

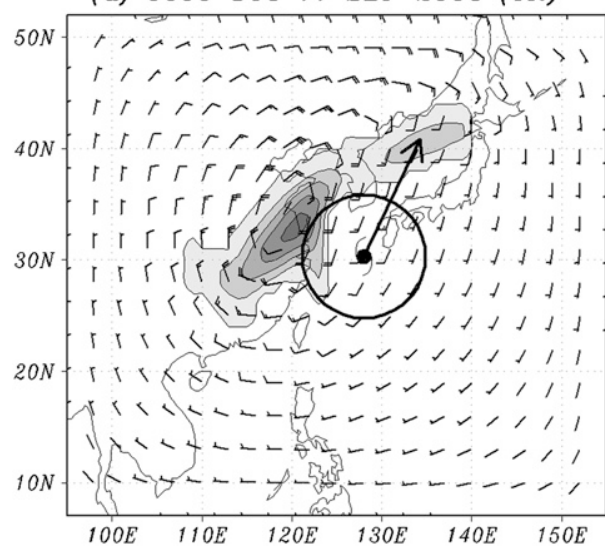

(b) 0000 UTC 17 SEP 2006 (SH)

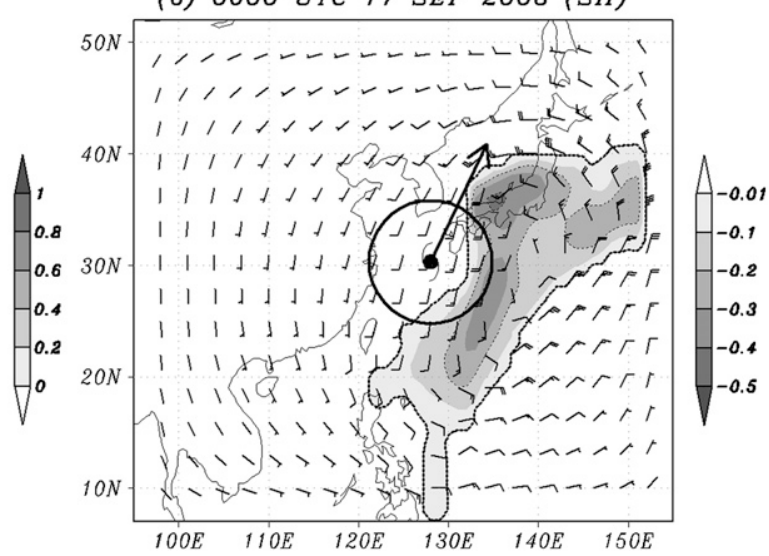

FIG. 7. Potential vorticity perturbation (scaled in PVU) at $500 \mathrm{hPa}$ and the balanced $925-300$-hPa deep-layer-mean steering wind (one full wind barb $=5 \mathrm{~m} \mathrm{~s}^{-1}$ ) associated with (a) the midlatitude trough $\left(q_{\mathrm{TR}}^{\prime}\right)$, and (b) the subtropical high $\left(q_{\mathrm{SH}}^{\prime}\right)$ at $0000 \mathrm{UTC} 17$ Sep 2006. The definitions of the arrow and circle are as in Fig. 2. 


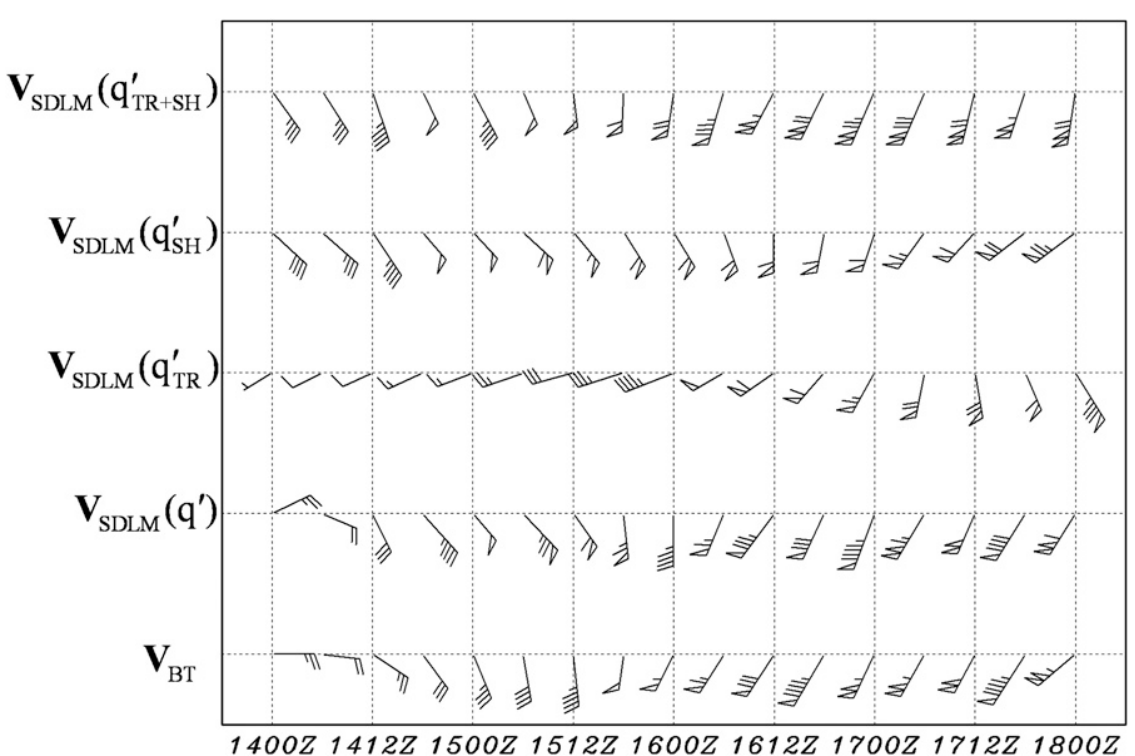

FIG. 8. The time series of the movement of Shanshan $\left(\mathbf{V}_{\mathrm{BT}}\right)$ and the steering flow (averaged in the inner $3^{\circ}$ latitude between 925 and $300 \mathrm{hPa}$ ) associated with the total PV perturbation $\left[\mathbf{V}_{\mathrm{SDLM}}\left(q^{\prime}\right)\right]$, and the PV perturbations of the trough $\left[\mathbf{V}_{\mathrm{SDLM}}\left(q_{\mathrm{TR}}^{\prime}\right)\right]$, the subtropical high $\left[\mathbf{V}_{\mathrm{SDLM}}\left(q_{\mathrm{SH}}^{\prime}\right)\right]$, and both of them $\left[\mathbf{V}_{\mathrm{SDLM}}\left(q_{\mathrm{TR}+\mathrm{SH}}^{\prime}\right)\right]$, respectively. One full wind barb (a flag) represents $1(5) \mathrm{m} \mathrm{s}^{-1}$

effect from both the trough and the subtropical high, as captured by the ADSSV $48 \mathrm{~h}$ prior to the verifying time.

\section{Summary}

Wu et al. (2007b) have proposed a new method (ADSSV) to demonstrate the sensitivity to the storm's steering flow. By appropriately defining the response functions to represent the mean steering flow at the verifying time, the sensitivity (i.e., ADSSV) to the flow field at the observing time can be obtained through the adjoint calculation. In the current study, three experiments starting from 0000 UTC 14, 15, and 16 September 2006, respectively, for Typhoon Shanshan are conducted using the MM5 adjoint modeling system to evaluate the typhoon-trough interaction from the ADSSV perspective.

Results show that in addition to the sensitivity located at the edge of the subtropical high (at about 800-500 $\mathrm{hPa}$ ) to the southeast of Shanshan, distinct ADSSV signals are present near the midlatitude trough over northern-central China. Evolution of the ADSSV also shows that the clear signal of sensitivity propagates along with the trough, implicating that the steering flow of Shanshan is likely to be affected by the flow associated with the trough at the verifying time (0000 UTC 17 September). On the other hand, the vertical structure of the ADSSV indicates that some sensitive signals associated with the midlatitude trough occur to the far northwest of Shanshan in the middle to upper tropo- sphere at about 500-300 $\mathrm{hPa}$, in good agreement with the physical intuition.

As indicated by the results of ADSSV, small vorticity perturbations associated with the trough at the observing time would likely lead to the track variation of Shanshan at the verifying time. Thus, PV diagnosis is applied to interpret and verify the physical meaning of the ADSSV signals. The PV analysis shows that the steering effect of the trough indeed becomes significant after around 1200 UTC 16 September, while the flow associated with the subtropical high remains important for steering Shanshan during its lifetime. This result is

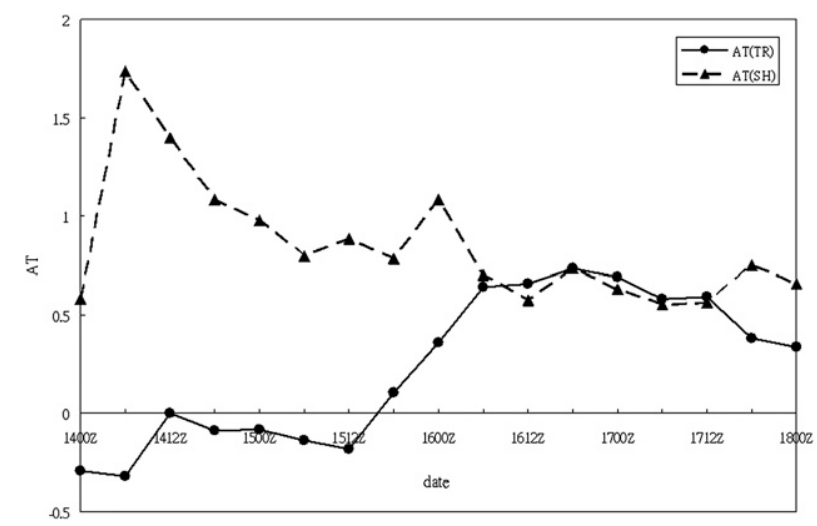

FIG. 9. The evolution of AT for the PV perturbation associated with the trough $\left(q_{\mathrm{TR}}^{\prime}\right.$; solid line) and the subtropical high $\left(q_{\mathrm{SH}}^{\prime}\right.$; dashed line) from 0000 UTC 14 to 18 Sep 2006. 
consistent with the ADSSV, which shows the sensitivity signals associated with both the trough and the edge of the subtropical high at the observing time (0000 UTC 15 September). On the other hand, the quantitative evaluation of the steering effect (AT) as in Wu et al. (2003) indicates that both the midlatitude trough and the subtropical high are dominant factors for the recurvature of Shanshan.

It is demonstrated that the ADSSV is able to pick up the large-scale trough features affecting the recurvature of Shanshan. Nevertheless, further verification and a sensitivity study of the ADSSV still need to be conducted. In particular, the PV field associated with the midlatitude trough or subtropical high could be perturbed to examine its response to the simulated typhoon track. In addition, PV diagnosis of data from model outputs can be performed to understand the balanced flows associated with PV perturbation in the region with distinct ADSSV values. Finally, note that several different targeted techniques [e.g., ADSSV, total energy singular vector (TESV; Peng and Reynolds 2006), ensemble transform Kalman filter (ETKF; Majumdar et al. 2002), and ensemble variance (Aberson 2003)] have been compared for the western North Pacific TCs in 2006 to identify the similarities and differences among all these different targeted methods and to interpret their meanings in terms of dynamics (Wu et al. 2008). The PV diagnosis conducted here can also provide very useful information to better understand the characteristics and dynamics of different targeted observation techniques and to improve the TC predictability.

Acknowledgments. The work is supported through the National Science Council of Taiwan by Grant NSC95-2119-M-002-039-MY2, the Office of Naval Research Grants N00014-05-1-0672 and N00173-08-1-G007, National Taiwan University Grant 97R0302, and the Central Weather Bureau Grant MOTC-CWB-96-6M-02.

\section{REFERENCES}

Aberson, S. D., 2003: Targeted observations to improve operational tropical cyclone track forecast guidance. Mon. Wea. Rev., 131, 1613-1628.

Charney, J. G., 1955: The use of primitive equations of motion in numerical prediction. Tellus, 7, 22-26.

Davis, C. A., 1992: Piecewise potential vorticity inversion. J. Atmos. Sci., 49, 1397-1411.

Errico, R. M., 1997: What is an adjoint model? Bull. Amer. Meteor. Soc., 78, 2577-2591.

Kim, H. M., and B.-J. Jung, 2006: Adjoint-based forecast sensitivities of Typhoon Rusa. Geophys. Res. Lett., 33, L21813, doi:10.1029/2006GL027289.
— and - 2009: Influence of moist physics and norms on singular vectors for a tropical cyclone. Mon. Wea. Rev., 137, 525-543.

Kleist, D. T., and M. C. Morgan, 2005: Interpretation of the structure and evolution of adjoint-derived forecast sensitivity gradients. Mon. Wea. Rev., 133, 466-484.

Langland, R. H., 2005: Issues in targeted observing. Quart. J. Roy. Meteor. Soc., 131, 3409-3425.

Majumdar, S. J., C. H. Bishop, B. J. Etherton, and Z. Toth, 2002: Adaptive sampling with the ensemble transform Kalman filter. Part II: Field program implementation. Mon. Wea. Rev., 130, 1356-1369.

Peng, M. S., and C. A. Reynolds, 2006: Sensitivity of tropical cyclone forecasts as revealed by singular vectors. J. Atmos. Sci., 63, 2508-2528.

Shapiro, L. J., 1996: The motion of Hurricane Gloria: A potential vorticity diagnosis. Mon. Wea. Rev., 124, 2497-2508.

Wu, C.-C., 2006: Targeted observation and data assimilation for tropical cyclone track prediction. Proc. Sixth Int. Workshop on Tropical Cyclones, San Jose, Costa Rica, WMO/CAS/ WWW, 409-423.

_ , and K. A. Emanuel, 1995a: Potential vorticity diagnostics of hurricane movement. Part I: A case study of Hurricane Bob (1991). Mon. Wea. Rev., 123, 69-92.

_ , and $-1995 \mathrm{~b}$ : Potential vorticity diagnostics of hurricane movement. Part II: Tropical Storm Ana (1991) and Hurricane Andrew (1992). Mon. Wea. Rev., 123, 93-109.

— understanding and future challenges. Bull. Amer. Meteor. Soc., 80, 67-80.

_- T.-S. Huang, W.-P. Huang, and K.-H. Chou, 2003: A new look at the binary interaction: Potential vorticity diagnosis of the unusual southward movement of Typhoon Bopha (2000) and its interaction with Typhoon Saomai (2000). Mon. Wea. Rev., 131, 1289-1300.

$\longrightarrow,-$, and K.-H. Chou, 2004: Potential vorticity diagnosis of the key factors affecting the motion of Typhoon Sinlaku (2002). Mon. Wea. Rev., 132, 2084-2093.

— , and Coauthors, 2005: Dropwindsonde Observations for Typhoon Surveillance near the Taiwan Region (DOTSTAR): An overview. Bull. Amer. Meteor. Soc., 86, 787-790.

—_, K.-H. Chou, P.-H. Lin, S. D. Aberson, M. S. Peng, and T. Nakazawa, 2007a: The impact of dropwindsonde data on typhoon track forecasts in DOTSTAR. Wea. Forecasting, 22, 1157-1176.

, J.-H. Chen, P.-H. Lin, and K.-H. Chou, 2007b: Targeted observations of tropical cyclone movement based on the adjointderived sensitivity steering vector. J. Atmos. Sci., 64, 2611-2626.

— , and Coauthors, 2008: Intercomparison of targeted observation guidances for tropical cyclones in the western North Pacific. Preprints, 28th Conf. on Hurricanes and Tropical Meteorology, Orlando, FL, Amer. Meteor. Soc., 7C.3.

Yang, C.-C., C.-C. Wu, K.-H. Chou, and C.-Y. Lee, 2008: Binary interaction between Typhoons Fengshen (2002) and Fungwong (2002) based on the potential vorticity diagnosis. Mon. Wea. Rev., 136, 4593-4611.

Zou, X., F. Vandenberghe, M. Pondeca, and Y.-H. Kuo, 1997: Introduction to adjoint techniques and the MM5 adjoint modeling system. NCAR Tech. Note NCAR/TN-435+STR, 110 pp. [Available from NCAR, P.O. Box 3000, Boulder, CO 80307-3000.] 